\title{
D-002 Ameliorates Histological Changes in Rats with Bile Reflux-Induced Esophagitis
}

\author{
Miriam $\mathrm{Noa}^{1, a^{*}}$, Rosa Mas ${ }^{2, b}$, Sarahí Mendoza ${ }^{3, \mathrm{c}}$, Zullyt Zamora $^{4, \mathrm{~d}}$, \\ Vivian Molina ${ }^{5, e}$, Maikel Valle, \\ ${ }^{1-6}$ Laboratory of Histopathology, Department of Pharmacology, Centre of Natural Products, \\ P. Box 6880, Havana, Cuba \\ a miriam.noa@cnic.edu.cu, ${ }^{b}$ rosa.mas@cnic.edu.cu, ${ }^{\mathrm{c}}$ sarahi.mendoza@cnic.edu.cu, \\ dzullyt.zamora@cnic.edu.cu, ${ }^{\mathrm{e}}$ vivian.molina@cnic.edu.cu, ${ }^{\mathrm{f}}$ maikel.valle@cnic.edu.cu
}

Keywords: beeswax alcohols, D-002, duodenoesophageal reflux, esophagitis, rats

\begin{abstract}
Reflux esophagitis, a common gastrointestinal disease, may be induced by duodeno gastroesophageal-reflux, and by duodeno-esophageal reflux (DER), the reflux of the biliary content only. D-002, a mixture of six higher primary alcohols purified from the beeswax, has exhibited gastric and esophageal protection experimentally. The aim of this work was to investigate the effects of D-002 on the esophageal histological changes in rats with DER-induced esophagitis. Rats were randomized into six groups: a negative control and five with DER: a positive control, three D-002- $(50,200$ and $400 \mathrm{mg} / \mathrm{kg}$, respectively) and one omeprazole $(20 \mathrm{mg} / \mathrm{kg})$ group, treated for 14 days. Microscopic studies of esophagus were performed looking for reflux esophagitis indicators, such as basal cell hyperproliferation, papillae elongation, inflammatory cell infiltrates, epithelial erosions and ulcerations. The thickness of esophageal epithelium was measured and leukocytes were counted. Histological characteristics of esophagitis were evaluated. Negative controls had normal esophagus, while positive controls exhibited marked esophagitis. The values of the histological score, thickness of the esophageal epithelium and leukocyte infiltration in the positive control group were higher $(\mathrm{p}<0.001)$ than in the negative control. D-002 $(50-400 \mathrm{mg} / \mathrm{kg})$ reduced significantly $(\mathrm{p}<0.01)$ the histological scores versus the positive control group. D-002 lowered significantly the epithelium thickness and abolished $(\mathrm{p}<0.001)$ leukocytes infiltration versus the positive control. Compared with the positive control, omeprazole reduced $(p<0.01)$ the histological score, the epithelium thickness and leukocytes infiltration. Repeated doses of D-002 ameliorated the histological changes in rats with DER-induced esophagitis.
\end{abstract}

\section{List of Abreviations:}

RE: Reflux esophagitis

LES: lower esophageal sphincter

DGER: duodenogastroesophageal-reflux

GER: gastroesophageal reflux

DER: duodeno-esophageal reflux

GERD: gastroesophageal reflux disease

\section{Introduction}

Reflux esophagitis (RE) is one of the commonest gastrointestinal diseases currently. RE results from the regurgitation of gastric content that ultimately is due to the malfunction of the lower esophageal sphincter (LES) that allows that gastric juice backs to the esophagus. Esophagitis may be induced by duodenogastroesophageal-reflux (DGER), the flow back of gastric, biliary and pancreatic contents into the stomach; and by duodeno-esophageal reflux (DER), the reflux of the biliary content only. [1 - 3] In patients with gastroesophageal reflux (GER), the concomitant reflux of duodenal content into the lower esophagus can cause mixed (alkaline and acid) RE that may lead to Barett's metaplasia and adenocarcinoma. [4 -6] 
Esophagus inflammation results from the unbalance between aggressive (mixed gastric acid and duodenal juice reflux) and defensive (esophageal acid clearance, esophageal tissue resistance) factors. [3] Short exposure of the esophagus to bile acids induce oxidative stress, [7, 8] mutations $[9,10]$ and apoptosis. [11] In turn, long-term exposure of esophageal tissue to bile reflux lead to apoptosis resistance and eventually to develop adenocarcinomas. [12, 13] In patients with gastroesophageal reflux disease (GERD), the concentration of bile acids in the oesophageal reflux is consistent with the degree of oesophageal mucosal damage. $[13,14]$

Management of GERD, a chronic disease, is complex, spontaneous remission is rare and life style modification is unlikely to relieve the symptoms. Majority of GERD require long-term acid suppressants, such proton pump inhibitors (PPI) (first-line choice) and $\mathrm{H} 2$ receptor antagonists (HsRA), but the end point of treatment is not clear. [15, 16] Nevertheless, there are a substantial number of poor responders to standard PPI doses due to transient LES relaxations, sensitivity to weakly acidic and/or alkaline reflux, large volume of reflux and/or esophageal hypersensitivity. [16] Also, a number of patients experience PPI-related adverse effects, such as Clostridium difficile colitis, osteoporosis, and vitamin B12 deficiency. [15 - 18]

D-002, a mixture of six higher primary alcohols purified from the beeswax, [19] has been shown to exert gastroprotection through multiple mechanisms. [20 - 24] Devoid of acid suppressant effect, $[20,21]$ the mechanisms of the gastroprotective action of D-002 involves the improved quality (content of proteins, glycoproteins, sulphated macromolecules) and increased secretion of gastric mucus, [21,22] anti-inflammatory and antioxidant effects on the gastric mucosa. [23,24] Oral administration of D-002 has demonstrated to reduce hydroxyl radicals, [21] malondialdehyde (MDA) $[23,24]$ and carbonyl groups concentrations, and myeloperoxidase (MPO) activity in the rat gastric mucosa, and to increase the activity of antioxidant enzymes (glutathione peroxidase, superoxide dismutase and catalase). [24] Also, the ability of D-002 for inhibiting 5-lipoxygenase (5-LOX) [25] and to lower concentrations of leukotriene-B4 (LTB4), a gastrotoxic mediator, [26] could contribute to its gastroprotective effects.

The beneficial effects of D-002 on the gastrointestinal system, however, involve another target beyond the reduction of gastric damage induced by several factors since esophagus-protective effects of D-002 have been demonstrated. [27, 28] Oral acute administration of D-002 reduced GER-induced esophageal lesions and increased oxidative stress in rats, without modifying gastric secretion acidity. [27] Also, repeat oral doses of D-002 (200 - $800 \mathrm{mg} / \mathrm{kg})$ ameliorated DERinduced esophagitis in rats, but failed to reduce DGER-induced esophagitis assessed by macroscopic evaluation of the lesions. [28]

In light of these facts, this study investigated whether D-002 was able to reduce microscopic esophagus lesions in rats with DER.

\section{Materials and Methods}

\section{Animals}

Male Sprague Dawley rats $(200$ - $250 \mathrm{~g})$ were purchased from the National Centre for Laboratory Animals Production (CENPALAB, Havana, Cuba) and adapted for 7 days to the following conditions: temperature $25 \pm 20 \mathrm{C}$, humidity $60 \pm 5 \%$ and light/dark cycles of $12 \mathrm{~h}$. Standard chow pellets from CENPALAB and water were given ad libitum. Rats were deprived of food for the $24 \mathrm{~h}$ prior to DER induction, but with free access to water.

Animal experiments were conducted in accordance with the Cuban Guidelines of Animals Handling and the Cuban Code of Good Laboratory Practices (GLP), which follow international guidelines for the use and care of laboratory animals. The study protocol and animals use were approved prior to the study by the Institutional Animal Ethics Committee.

\section{Chemicals and test substance}

The batch of D-002, supplied by the Plants of Natural Products (Havana, Cuba), had the following composition: tetracosanol (7.0\%), hexacosanol (11.5\%), octacosanol (12.1\%), 
triacontanol (34.8\%), dotriacontanol (22.5\%) and tetratriacontanol (2.6\%). Purity (total content of these alcohols) was $90.0 \%$. Omeprazole was purchased from DOMER (Mexico).

\section{Dosage and administration}

The rats used in this study were those of a previous study that investigated the effects of D002 on the esophageal lesions index (ELI) and macroscopic esophageal findings of esophagitis in rats with DER. In brief, D-002 and omeprazole were suspended in a 1\% acacia gum/water. Rats were randomized into six groups of 8 rats each: a negative vehicle control and five with DER: a positive vehicle control, three treated with D-002 (50, 200 and $400 \mathrm{mg} / \mathrm{kg}$, respectively), one with omeprazole $20 \mathrm{mg} / \mathrm{kg}$. All treatments (D-002, omeprazole, or vehicle) were given orally by gastric gavage $(1 \mathrm{~mL} / 200 \mathrm{~g}$ of bodyweight), every $24 \mathrm{~h}$ during 14 days before DER induction. The last administration happened 24 hours before animals' sacrifice.

The chosen doses of D-002 were those effective in the model of gastroesophageal reflux (GER)-induced esophagitis in rats, [27, 28] while that of omeprazole has been reported as effective in a GER model in rats. [29]

\section{DER-induced esophagitis in rats}

DER was surgically induced in rats according to Hashimoto 2012. [30] Solid foods was withdrawn 1 day before and for 1 day after surgery. The esophagus-duodenum anastomosis was performed under general anaesthesia (thiopental $30 \mathrm{mg} / \mathrm{kg}$ body wt ip) through an upper midline incision. The gastroesophageal junction was then ligated and the distal esophagus was transected $2 \mathrm{~mm}$ above the ligature. In addition, the gastroduodenal junction was ligated and the proximal duodenum was transected $3 \mathrm{~mm}$ distal to the pylorus. A total gastrectomy was performed with the removal of the entire stomach and end-to-end anastomosis of the esophagus and duodenum was done. The abdominal incision was closed in two layers. The animals were sacrificed on day 14 after the operation. The esophagus was removed, incised lengthwise and processed for microscopic studies.

\section{Microscopic study}

Esophagus were washed with $0.9 \% \mathrm{NaCl}$ saline and fixed in $10 \%$ buffered formaldehyde. Samples from the lower $2 / 3$ of the esophagus, including the duodenum esophageal junction (DEJ), were taken and subsequently embedded in paraffin. Sections were stained with haematoxylin and eosin and examined using a Carl Zeiss Primo Star microscope.

Sections were examined looking for reflux esophagitis indicators, such as inflammatory cell infiltrates, basal cell hyperproliferation, papillae elongation, dilated venules, increased capillaries, epithelial erosions and ulcerations. [31, 32]

The thickness of esophageal epithelium was measured randomly in 10 areas per animal. Also, leukocytes were counted in ten random areas of the esophageal mucosa, with higher magnification (400 X).

Histological characteristics of esophagitis were evaluated according to the Hetzel-Dent scoring system, as described by Aiyer, 2011. [33]

1. Normal appearance of the mucosa

2. Basal cell hyperplasia, edematous mucosa, hyperemia, friability of the mucosa

3. Surface erosion involving $<10 \%$ of the mucosal surface of the last $5 \mathrm{~cm}$ of the esophageal squamous mucosa

4. Superficial erosions or ulcerations involve between $10-50 \%$ of the surface of the mucosa.

5. Deep ulceration of $>50 \%$ of esophageal squamous mucosa in five random regions for each sample. The average score for each group was calculated.

\section{Statistical Analysis}

Paired comparisons between treated and control groups were done by using the Mann Whitney $U$ test. Categorical data (number of animals with esophagitis, dysplasia and inflammatory infiltrate) were compared with the Fischer Exact Probability test. All tests were two tailed. The 
level of statistical significance was set at $\alpha=0.05$. Analyses were done by using the Statistic software for Windows (Release 4.2, Stat Soft, Inc USA).

\section{Results}

All negative control rats exhibited normal esophagus (score 1) lined by keratinized stratified plane epithelium with 4-7 epithelial cell layers, and presenting the keratin with saw appearance. Beneath the epithelium there was a thin layer, comprising smooth muscle separating the lamina propria of the submucosa, with interruptions (muscularis mucosae). The muscular layer was divided in two strata (Fig. 1).

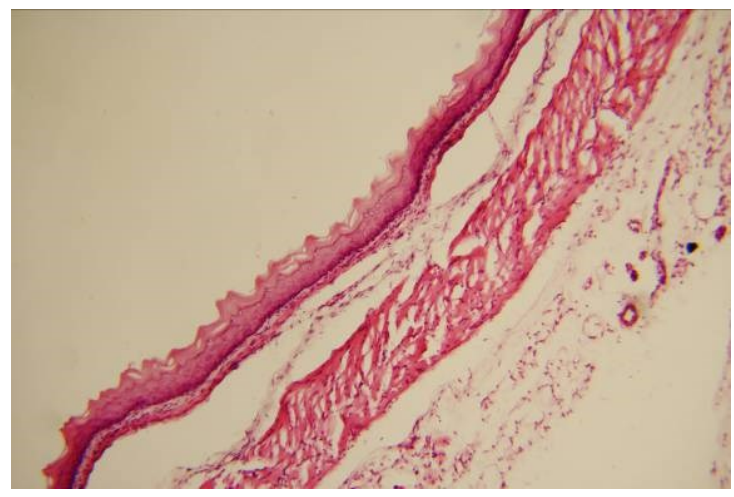

Figure 1. Normal esophagus lined by keratinized stratified plane epithelium presenting the keratin with saw appearance. 40X HE

By the opposite, all positive controls displayed marked signs of esophagitis (score 4) present in almost the entire length of the esophagus, but not milder lesions, such as hyperplasia or erosions $<10 \%$ (scores 2 and 3). Esophagitis was characterized by a markedly thickened stratified epithelium with basal cell hyperplasia and acanthosis, hyperkeratosis, and elongation of the papillae of the lamina propria into the epithelium. Some hyperplastic changes had areas of inverted endophytic papillomatosis, with verrucous aspect by the presence of buds type. The squamous epithelium had wide continuity solutions, erosions, ulceration, and large areas of inflammatory infiltrates affecting mainly the lamina propria and submucosa, but also extended to the muscular layer, exhibiting the presence of polymorphonuclear leukocytes, eosinophils and mast cells. Changes were also observed in the arteries, with hypertrophy of the medial muscular layer and consequent thinning of lumen, with vacuolization of the vascular endothelium (Fig. 2 and Fig. 3).

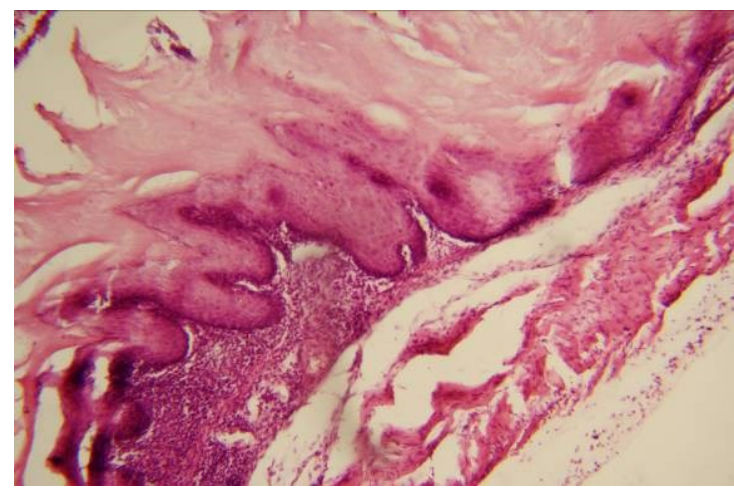

a

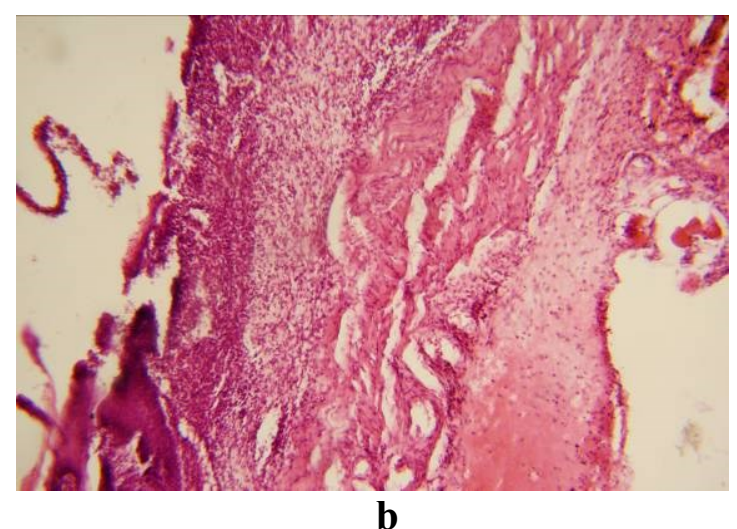

b

Figure 2. a: Positive control showing extensive esophagitis with markedly thickened stratified epithelium and basal cell hyperplasia, hyperkeratosis, elongation of the papillae of the lamina propria and inflammatory infiltrate; b: Positive control with extensive ulceration and inflammatory infiltrate. 40X HE 
The frequency of animals with esophageal abnormalities corresponding to score 4 (erosions / ulcerations 10-50\%) in the DER positive control group was higher than in the negative control, while the groups treated with D-002 $(50-400 \mathrm{mg} / \mathrm{kg})$ or OMP $20 \mathrm{mg} / \mathrm{kg}$ had lower frequencies of rats with score 4 as compared to the positive control $(\mathrm{p}<0.01)$. D-002 treated rats $(50-400 \mathrm{mg} / \mathrm{kg})$, however, exhibited minor abnormalities such as hyperplasia as compared to the control groups (Tables 1 and 2). The highest score (5) (ulceration in over 50\% of the esophageal mucosa) was not seen in any animal. In line with these results, the average histological score in the positive control group was significantly higher $(\mathrm{p}<0.01)$ than in the negative control, while OMP $20 \mathrm{mg} / \mathrm{kg}$, the reference substance, reduced such values significantly as compared to the positive control group. Likewise, oral treatment with D-002 $(50,200$ and $400 \mathrm{mg} / \mathrm{kg})$ also decreased significantly $(\mathrm{p}<0.01)$ the histological scores versus the positive control values. Fig 3, 4 and 5.

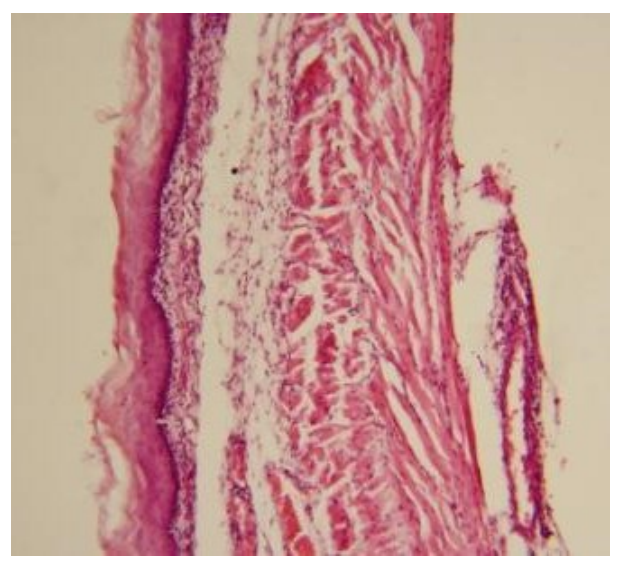

Figure 3. D-002 $50 \mathrm{mg} / \mathrm{Kg}$

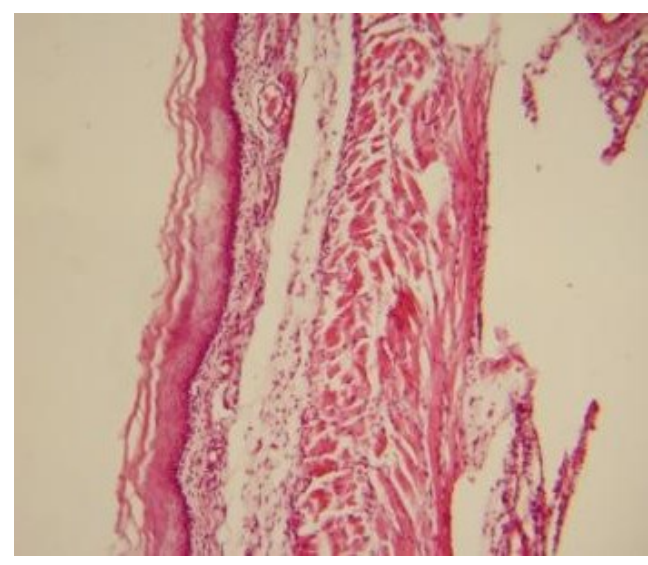

Figure 4. D-002 200 mg/Kg

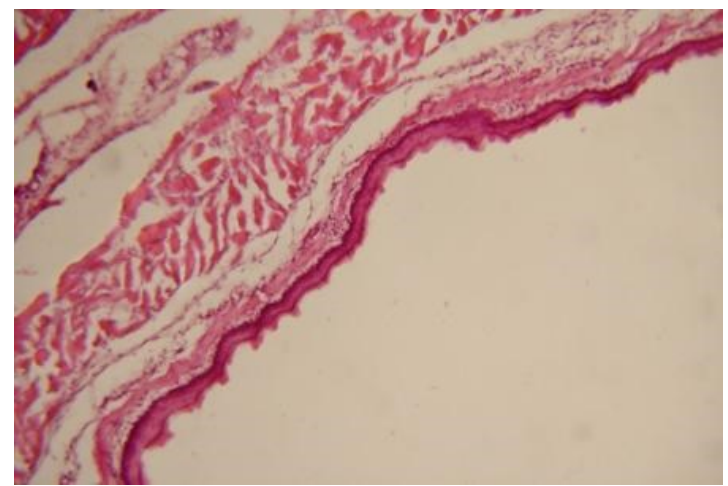

Figure 5. D-002 $400 \mathrm{mg} / \mathrm{Kg}$

Figures 3, 4, 5: D-002 treatment groups showing minor abnormalities. 40X HE

Table 1. Distribution of esophagitis histological lesions in rats with bile reflux-induced by DER

\begin{tabular}{|c|c|c|c|c|c|}
\hline Groups & $\begin{array}{c}\text { Normal } \\
\mathbf{( 1 )}\end{array}$ & $\begin{array}{c}\text { Hyperplasia } \\
\mathbf{( 2 )}\end{array}$ & $\begin{array}{c}\text { Erosions } \\
<\mathbf{1 0 \%} \text { (3) }\end{array}$ & $\begin{array}{c}\text { Erosions or } \\
\text { Ulcerations } \\
\mathbf{1 0}-\mathbf{5 0 \%} \text { (4) }\end{array}$ & $\begin{array}{c}\text { Ulceration } \\
\mathbf{5 0 \%} \text { (5) }\end{array}$ \\
\hline $\begin{array}{c}\text { Negative control } \\
\text { (Vehicle + sham) }\end{array}$ & $8 / 8^{* * *}$ & $0 / 8$ & $0 / 8$ & $0 / 8^{* * *}$ & $0 / 8$ \\
\hline $\begin{array}{c}\text { Positive Control } \\
\text { (Vehicle + DER) }\end{array}$ & $0 / 8$ & $0 / 8$ & $0 / 8$ & $8 / 8$ & $0 / 8$ \\
\hline D-002 50 mg/kg + DER & $0 / 8$ & $5 / 8^{*}$ & $3 / 8$ & $0 / 8^{* * *}$ & $0 / 8$ \\
\hline D-002 200 mg/kg + DER & $2 / 8$ & $5 / 8^{*}$ & $1 / 8$ & $0 / 8^{* * *}$ & $0 / 8$ \\
\hline D-002 400 mg/kg + DER & $1 / 7$ & $6 / 7^{* *}$ & $0 / 7$ & $0 / 7^{* * *}$ & $0 / 7$ \\
\hline OMP 20 mg/kg + DER & $2 / 8$ & $2 / 8$ & $3 / 8$ & $1 / 8^{* *}$ & $0 / 8$ \\
\hline
\end{tabular}

Histological lesions were assessed by using the Hetzel-Dent scoring system,

DER duodeno-esophageal reflux, OMP Omeprazole $* \mathrm{P}<0.05 ; * * \mathrm{p}<0.01 ; * * * \mathrm{p}<0.001$. Comparisons with the positive DER control (Fischer Test) 
Table 2. Histological scores in rats with bile reflux induced by DER in rats

\begin{tabular}{|c|c|c|}
\hline Groups & Scores & \% inhibition \\
\hline Negative control (Vehicle + sham) & $1.00 \pm 0.00^{* *}$ & \\
\hline Positive Control (Vehicle + DER) & $4.00 \pm 0.00$ & \\
\hline D-002 50 $\mathbf{~ m g / k g ~ + ~ D E R ~}$ & $2.38 \pm 0.52^{* *}$ & $\mathbf{5 4 . 0}$ \\
\hline D-002 200 $\mathbf{~ m g / k g ~ + ~ D E R ~}$ & $1.88 \pm 0.64^{* *}$ & $\mathbf{7 0 . 7}$ \\
\hline D-002 400 $\mathbf{~ m g / k g ~ + ~ D E R ~}$ & $1.88 \pm 0.35^{* *}$ & $\mathbf{7 0 . 7}$ \\
\hline OMP 20 $\mathbf{~ m g / k g ~ + ~ D E R ~}$ & $2.38 \pm 1.06^{*}$ & $\mathbf{5 4 . 0}$ \\
\hline
\end{tabular}

Histological lesions were assessed by using the Hetzel-Dent scoring system,

DER duodeno-esophageal reflux, OMP Omeprazole

* $\mathrm{P}<0.01$; ** $\mathrm{p}<0.001$. Comparisons with the positive DER control (Mann Whitney U test) Dysplasia, defined by increased nuclear/cytoplasmic ratio, with irregular nuclei hyperchromatic, and nuclear pseudostratification, was seen in the esophageal epithelium of 2 positive control rats only. An architectural dysplasia was added to the nuclear dysplasia. No significant differences were seen in any comparison.

The thickness of esophageal epithelium and leukocyte infiltration in the positive control group was significantly higher than in the negative control $(\mathrm{p}<0.001)$ (Table 3$)$. Oral administration of D-002 (50, 200 and $400 \mathrm{mg} / \mathrm{kg})$ and OMP $20 \mathrm{mg} / \mathrm{kg}$ lowered significantly, but modestly, the epithelium thickness. D-002-induced inhibition of leukocytes infiltration was not only significant ( $\mathrm{p}$ $<0.001$ ) but complete, since D-002 50, 200 and $400 \mathrm{mg} / \mathrm{kg}$ produced inhibitions of $99.0 \%, 99.4 \%$ and $100 \%$ versus the positive control. OMP $20 \mathrm{mg} / \mathrm{kg}$ lowered leukocytes infiltration significantly $(\mathrm{p}<0.01)$ and markedly $(89.9 \%)$ but less than D-002 (p<0.05) (Fig. 6).

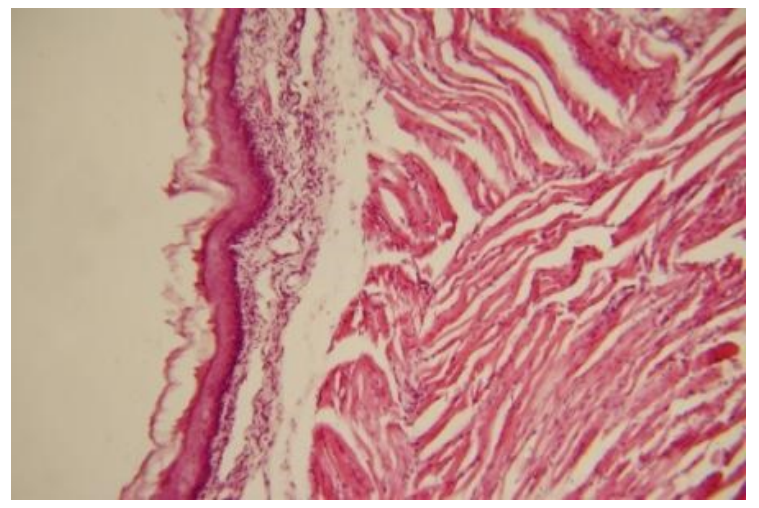

OMP $20 \mathrm{mg} / \mathrm{Kg}$

Figure 6. Omeprazole treatment group showing minor abnormalities.

Table 3. Epithelial thickness and leukocytes on the esophagus of rats with bile reflux induced by DER

\begin{tabular}{|c|c|c|c|c|}
\hline Groups & $\begin{array}{c}\text { Epitelial thickness } \\
(\boldsymbol{\mu m})\end{array}$ & \% I & Leukocytes (\#) & \% I \\
\hline Negative control (Vehicle + sham) & $25.80 \pm 0.29^{* *}$ & & $1.59 \pm 0.08^{* *}$ & \\
\hline Positive Control (Vehicle + DER) & $202.55 \pm 1.04$ & & $26.56 \pm 0.61$ & \\
\hline D-002 50 $\mathbf{~ g} / \mathbf{k g}+$ DER & $180.78 \pm 9.14^{* *}$ & $\mathbf{1 2 . 3}$ & $1.85 \pm 0.39^{* *+}$ & $\mathbf{9 9 . 0}$ \\
\hline D-002 200 $\mathbf{~ m g / k g}+$ DER & $155.84 \pm 1.60^{* *}$ & $\mathbf{2 6 . 4}$ & $1.74 \pm 0.27^{* *+}$ & $\mathbf{9 9 . 4}$ \\
\hline D-002 400 $\mathbf{~ m g / k g ~ + ~ D E R ~}$ & $160.19 \pm 9.19^{* *}$ & $\mathbf{2 4 . 0}$ & $1.57 \pm 0.31^{* *+}$ & $\mathbf{1 0 0}$ \\
\hline OMP 20 $\mathbf{~ m g / k g ~ + ~ D E R ~}$ & $162.83 \pm 1.76^{* *}$ & $\mathbf{2 2 . 5}$ & $4.11 \pm 0.68^{* *}$ & $\mathbf{8 9 . 9}$ \\
\hline
\end{tabular}

DER duodeno-esophageal reflux, OMP Omeprazole

$* \mathrm{P}<0.01 ; * * \mathrm{p}<0.001$. Comparisons with the positive DER control (Mann Whitney U test)

$+\mathrm{P}<0.001$. D-002 vs OMP (Mann Whitney U test) 


\section{Discussion}

The present study demonstrates, by the first time, the protective effect of oral administration of D- $002(50-400 \mathrm{mg} / \mathrm{kg})$ on the histological changes in the esophagus of rats with DER.

Since the positive control group exhibited all characteristics of esophagitis and higher values of histological scores, epithelium thickness and leukocytes infiltration than the negative control group and that OMP $20 \mathrm{mg} \mathrm{kg}$ (reference substance) ameliorated all these abnormalities confers validity to our experimental conditions and indicates that the effects of D-002 here reported were attributable to the treatment. This is reinforced by the fact that the distribution pattern of the lesion scores matches well with the histological scores, epithelium thickening and leukocyte infiltration in the different groups.

Oral treatment with D-002 $(50-400 \mathrm{mg} / \mathrm{kg})$ demonstrated an esophageal protective effect at microscopical level since produced significant reductions of the frequency of animals showing score 4 lesions and a marked decrease of the average histological score. The effect on this score, however, was not dose dependent, since the effects of 200 and $400 \mathrm{mg} / \mathrm{kg}$ were the same. Bearing in mind that the effect of the lowest dose tested $(50 \mathrm{mg} / \mathrm{kg})$ were marked, the inclusion of lower dose could have render the expected dose- effect relationship, but this rationale is just speculative.

In addition, D-002 $(50-400 \mathrm{mg} / \mathrm{kg})$ was effective for reducing significantly the esophageal epithelium thickening, a finding that may results from the DER-induced inflammatory infiltrates caused by the triggering release of proinflammatory cytokines. [34] This effect of D-002, however, was modest (12.3\% - 24.0\% inhibitions versus the DER positive control group), particularly if we acknowledge the marked effects on the histological score (about $70 \%)$ and the abolishment $(100 \%$ inhibition) of leukocyte infiltration (a marker of inflammation). It should be noted that such difference was also observed for the OMP group, in which histological score and leukocyte infiltration were reduced by $54.0 \%$ and $89.9 \%$, respectively, versus the positive control, while epithelium thickening lowered by $22.5 \%$. We don't find a conclusive reason to explain why both D002 and OMP are less effective for lowering epithelium thickening than for reducing the other indicators, but the effects of both treatments were grossly similar. The effect of D-002 200 and $400 \mathrm{mg} / \mathrm{kg}$ on leukocyte infiltration was greater than that OMP $20 \mathrm{mg} / \mathrm{kg}$, but since only one dose of OMP was tested we cannot conclude that D-002 was more effective than OMP on such regard. These results should not be surprising since both D-002 [24] and OMP [35] have shown to produce anti-inflammatory effects on the gastric mucosa.

The present results generally agree with the esophagus protective effects of D-002 reported previously. [27, 28] Firstly, a study demonstrated that acute oral administration of D-002 decreased macroscopic esophageal lesions and oxidative stress in rats with experimentally induced-GER, without modifying gastric secretion acidity. [27] Consistently, a second study found that repeat doses of D-002 (50, 200 and $400 \mathrm{mg} / \mathrm{kg})$ administered for 14 days before DER induction lowered significantly the esophageal lesions index (ELI) (to 59.2\%) and macroscopic inflammation (to $77.8 \%$ ) as compared to the DER positive control group. That study, however, found that D-002 was not effective for decreasing significantly macroscopic esophagitis changes, despite the higher doses (200 and $400 \mathrm{mg} / \mathrm{kg}$ ) produced apparent, but not significant decreases of such changes. [28] Our results seem to contradict this last result, but such a difference may be explained by the fact that the previous study assessed the changes macroscopically, and the present data were obtained by microscopic examination and evaluation, the definitive word for judging the occurrence of esophagitis.

The mechanisms whereby D-002 prevented the microscopic changes characteristics of esophagitis are beyond the scope of this study, which was focused to confirm the esophageal protective effect of D-002 at histological level. Nevertheless, in light of the role of the oxidative damage are critical in pathogenesis of RE [36] the antioxidant effects of D-002 [23, 24, 27] may contribute to the present results. In such regard, previous studies have demonstrated that the efficacy of D-002 for preventing indomethacin-induced gastric ulcers in rats was associated to the reduction of lipid peroxidation, $[23,24]$ protein oxidation, generation of hydroxyl radical and to the increase of superoxide dismutase and glutathione peroxidase enzyme activities in gastric mucosa. 
[24] Likewise, the esophageal protection of the acute administration of D-002 on rats with DERinduced esophageal damage was associated with a decrease of lipid peroxidation and protein oxidation in the esophageal tissue. [27] In this way, it is plausible to assume that the antioxidant effects of D-002 may have contributed, at least in part, to its efficacy in this model.

In addition, the anti-inflammatory effects of D-002, able to decrease the enzyme activity of myeloperoxidase (MPO) in the gastric mucosa, [24] could be also present on this target. Taking into account the ability of D-002 for inhibiting both cyclooxygenase (COX) and 5-lipoxygenase (5LOX) enzyme activities, the dual anti-inflammatory effect derived from the inhibition of both enzymes may also contribute to its esophagus protective effects.

Disregarding the mechanisms that support its action, the present results add new clues of the esophagus protective effect of D-002 evidenced microscopically, and extend the gastroprotection conferred by oral treatment with D-002 beyond the gastric mucosa.

\section{Conclusion}

The present data indicate that oral treatment with repeat doses of D-002 $(50-400 \mathrm{mg} / \mathrm{kg})$ during 14 days before DER ameliorated the histological changes in esophagus of rats with DER, since produced significant reductions of the frequency of animals showing score 4 lesions. The frequency of animals with esophageal abnormalities corresponding to score 4 (erosions/ulcerations $10-50 \%)$ in the DER positive control group was higher than in the negative control, while the groups treated with D-002 $(50-400 \mathrm{mg} / \mathrm{kg})$ or OMP $20 \mathrm{mg} / \mathrm{kg}$ had lower frequencies of rats with score 4 as compared to the positive control $(\mathrm{p}<0.01)$. D-002 treated rats $(50-400 \mathrm{mg} / \mathrm{kg})$, was effective exhibiting minor abnormalities such as hyperplasia as compared to the control groups and reducing significantly the esophageal epithelium thickening, a finding that may results from the DER-induced inflammatory infiltrates. D-002 induced inhibition of leukocytes infiltration, it was not only significant $(\mathrm{p}<0.001)$ but complete, since D-002 $(50,200$ and $400 \mathrm{mg} / \mathrm{kg})$ produced inhibitions of $99.0 \%, 99.4 \%$ and $100 \%$ versus the positive control. Omeprazole reduced also $(\mathrm{p}$ $<0.01)$ the histological score, the epithelium thickness. The effect of D-002 200 and $400 \mathrm{mg} / \mathrm{kg}$ on leukocyte infiltration was greater than that OMP $20 \mathrm{mg} / \mathrm{kg}(\mathrm{p}<0.05)$.

\section{Acknowledgments}

Thanks to Nilda Mendoza, for her technical assistance.

\section{References}

[1] J.Y. Mabrut, J.M. Collard, J. Baulieux, Duodenogastric and gastroesophageal bile reflux, J. Chir. 143(6) (2006) 355-365.

[2] A. Agrawal et al., Symptoms with acid and nonacid reflux may be produced by different mechanisms, Dis. Esophagus. 22 (2009) 467-470.

[3] G. Boeckxstaens et al., Symptomatic reflux disease: the present, the past and the future, Gut. 63(7) (2014) 1185-1193.

[4] S. Jürgens et al., The role of bile acids in the neoplastic progression of Barrett's esophagus - a short representative overview, Z. Gastroenterol. 50 (2012) 1028-1034.

[5] F. Hvid-Jensen et al., Incidence of adenocarcinoma among patients with Barrett's esophagus. N Engl J Med. 365 (2011) 1375-1383.

[6] K.R. McQuaid et al., Systematic review: the role of bile acids in the pathogenesis of gastrooesophageal reflux disease and related neoplasia, Aliment. Pharmacol. Ther. 34(2) (2011) 146-165. 
[7] Y.J. Kim, E.H. Kim, K.B. Hahm, Oxidative stress in inflammation-based gastrointestinal tract diseases: challenges and opportunities, J. Gastroenterol. Hepatol. 27 (2012) 1004-1010.

[8] K. Dvorak et al., Bile acids in combination with low $\mathrm{pH}$ induce oxidative stress and oxidative DNA damage: relevance to the pathogenesis of Barrett's oesophagus, Gut. 56 (2007) 763771.

[9] A.J. Jolly, C.P. Wild, L.J. Hardie. Sodium deoxycholate causes nitric oxide mediated DNA damage in oesophageal cells, Free Radic. Res. 43 (2009) 234-240.

[10] J. Theisen et al., The mutagenic potential of duodenoesophageal reflux, Ann Surg. 241 (2005) $63-68$

[11] R. Zhang et al., Bile salts inhibit growth and induce apoptosis of culture human normal esophageal mucosal epithelial cells, World J. Gastroenterol. 11 (2005) 6466-6471.

[12] K. Dvorakova et al., Apoptosis resistance in Barrett's esophagus: ex vivo bioassay of live stressed tissues, Am. J. Gastroenterol. 100 (2005) 424-431.

[13] T.K. Desai et al., The incidence of oesophageal adenocarcinoma in non-dysplastic Barrett's oesophagus: a meta-analysis, Gut. 61 (2012) 970-976.

[14] A. Nakos et al., The histological and immunohistochemical aspects of bile reflux in patients with gastroesophageal reflux disease, Gastroenterol. Res. Pract. 2011 (2011) 1-9.

[15] P.J. Kahrilas, N.J. Shaheen, M.F. Vaezi, American Gastroenterological Association medical position statement on the management of gastroesophageal reflux disease, Gastroenterol. 135 (2008) 1392-1413.

[16] D.A. Johnson, B.H. Levy, 3rd Evolving drugs in gastroesophageal reflux disease: pharmacologic treatment beyond proton pump inhibitors, Expert Opinion on Pharmacotherapy. 11(9) (2010) 1541-1548.

[17] J. Venkataraman, A. Krishnan, Long-term medical management of gastro-esophageal reflux disease: how long and when to consider surgery?, Trop. Gastroenterol. 33(1) (2012) 21-32.

[18] S.M. Wilhelm, R.G. Rjater, P.B. Kale-Pradhan, Perils and pitfalls of long-term effects of proton pump inhibitors, Expert Rev. Clin. Pharmacol. 6 (2013) 443-451.

[19] Mas R. D-002: A product obtained from beeswax, Drugs of the Future. 26 (2001) 731-744.

[20] D. Carbajal et al., Anti-ulcer activity of higher primary alcohols of beeswax, J. Pharm. Pharmacol. 47 (1995) 731-733.

[21] D. Carbajal et al., Possible cytoprotective mechanism in rats of D-002 an anti-ulcerogenic product isolated from beeswax, J. Pharm. Pharmacol. 48 (1996) 858-860.

[22] D. Carbajal et al., Effects of D-002 on gastric mucus composition in ethanol-induced ulcer, Pharmacol. Res. 42 (2000) 329-332.

[23] V. Molina et al., Antioxidant effects of D-002 on gastric mucosa of rats with experimentallyinduced injury, J. Med. Food. 4 (2001) 79-83.

[24] Y. Pérez et al., Protective effect of D-002, a mixture of beeswax alcohols, against indomethacin-induced gastric ulcers and mechanism of action, J. Nat. Med. 67 (2013) 182189.

[25] Y. Pérez et al., Inhibition of cyclooxygenase and 5-lipooxygenase enzymes by D-002 (beeswax alcohols). Current Topics in Nutraceuticals Research. 12(1/2) (2014) 13-18.

[26] M. Okazaki et al., Gastric mucosal levels of prostaglandins and leukotrienes in patients with gastric ulcer after treatment with rabeprazole in comparison to treatment with ranitidine, J. Med. Invest. 54 (2007) 83-90 
[27] Z. Zamora et al., Protective effects of D-002 on experimentally induced gastroesophageal reflux in rats, World J. Gastroenterol. 20 (2014) 2085-2090.

[28] Z. Zamora et al., Effects of D-002 on esophagitis induced by duodenal- esophageal reflux (DER) and duodenogastro-esophageal reflux (DGER) in rats, Int. J. of Pharmacol. Toxicol. 5(3) (2015) 146-151.

[29] N. Inatomi et al., Effects of a proton pump inhibitor, AG-1749 (Lanzoprazole), on reflux esophagitis and experimental ulcers in rats, Japan J. Pharmacol. 55 (1991) 437-451.

[30] N. Hashimoto, Expression of COX2 and p53 in rat esophageal cancer induced by reflux of duodenal contents, ISRN Gastroenterol. 2012 (2012) 1-5.

[31] Y. Li, R.C. Martin, 2nd Reflux injury of esophageal mucosa: experimental studies in animal models of esophagitis, Barrett's esophagus and esophageal adenocarcinoma, Dis. Esophagus. 20 (2007) 372-378.

[32] H. Aiyer, L. Yan, R. Martin, Diet composition affects surgery-associated weight loss in rats with a compromised alimentary tract, J. Surg. Res. 168 (2011) 42-48.

[33] H.S. Aiyer et al., Dietary freeze dried black raspberry's effect on cellular antioxidant status during reflux-induced esophagitis in rats, Nutrition. 27 (2011) 182-187.

[34] D. Asaoka et al., Characteristic pathological findings and effects of ecabet sodium in rat reflux esophagitis, World J. Gastroenterol. 15(28) (2009) 3480-3485.

[35] R.R. Kedika, R.F. Souza, S.J. Spechler, Potential anti-inflammatory effects of proton pump inhibitors: a review and discussion of the clinical implications, Digestive diseases and sciences. 54(11) (2009) 2312-2317.

[36] T.Y. Oh et al., Oxidative damages are critical in pathogenesis of reflux esophagitis: implication of antioxidants in its treatment, Free Radic Biol Med. 30 (2001) 905-915. 